\title{
MEASURING OCEAN WAVES FROM SPACE: OBJECTIVES AND CHARACTERISTICS OF THE CHINA-FRANCE OCEANOGRAPHY SATELLITE (CFOSAT)
}

\author{
Lefèvre Jean-Michel \\ Météo France \\ Toulouse, France \\ Aouf Lotfi \\ Météo France \\ Toulouse, France
}

Hauser Danièle

UVSQ, CNRS, UPMC

LATMOS, 10- 12 Avenue de l'Europe

78140- Vélizy, France

\author{
Tison Céline \\ CNES , "Altimetry and Radar office" \\ 18 avenue Edouard Belin \\ 31400 Toulouse, France
}

\author{
Lambin Juliette \\ CNES \\ Toulouse, France \\ Collard Fabrice \\ CLS \\ Brest, France
}

\author{
Thierry Amiot \\ CNES \\ Toulouse, France \\ Castillan Patrick \\ CNES \\ Toulouse, France
}

\begin{abstract}
The Chinese and French Space Agencies are jointly preparing a satellite mission devoted to the monitoring of the ocean surface and related science and applications. This is the so-called "China France Oceanography SATellite" (CFOSAT), to be launched around 2013. This mission will provide simultaneous and collocated observations of wind at the ocean surface and spectral properties of surface ocean waves using two scatterometers, both in Ku-Band: SWIM for measurements of directional wave spectra and SCAT for wind vector measurements. The SWIM instrument will use a real aperture observation technique so as to avoid limitations encountered with SAR systems. This paper describes the main objectives and characteristics of the mission with a focus on the SWIM instrument designed and developed under French responsibility to measure directional spectra of ocean waves.
\end{abstract}

\section{THE CFOSAT MISSION}

Oceanography greatly benefits from remote sensing satellites for global monitoring and forecast of the sea state. The CFOSAT (China France Oceanography SATellite) mission, whose launch is planned for 2013, is designed to provide at the global scale, observations of wind at the ocean surface and spectral properties of surface ocean waves. It will serve both operational needs for the surface wind and wave forecast (marine meteorology and climatology), and research needs by improving our knowledge on waves hydrodynamics, on interactions between waves and the atmospheric or oceanic boundary layers, and on interactions between electromagnetic signals and the ocean surface.

The CFOSAT mission consists of a polar orbiting system at about $514 \mathrm{~km}$ altitude. The platform will embark two payloads, both are radar at $\mathrm{Ku}$-band $(13.2$ to $13.6 \mathrm{GHz})$ scanning around the vertical axis; one is SWIM 'Surface Waves Investigation and Monitoring)', which is a 6-beam radar at small incidence $\left(0\right.$ to $\left.10^{\circ}\right)$, with the nadir pointing beam providing like standard altimeter missions wind speed and significant wave height and the 6 to $10^{\circ}$ beams devoted to the measurement of directional spectra of ocean waves; it is a wave scatterometer; the other one is SCAT which is a rotating fanbeam wind-scatterometer covering the $20-60^{\circ}$ incidence angles. This combination of small to medium incidence angles is appropriate to measure simultaneously the wind vector (from large incidence) and wave properties (from small incidence). The combination of the two instruments will help to separate the different effects governing the evolution of the wave field.

CFOSAT will provide at the global scale, observations of wind vector, directional energy spectra of ocean waves (with wavelength between 70 and $500 \mathrm{~m}$ ), significant wave height, steepness of long waves, and parameters characterizing the statistics of wave slopes (for waves with wavelength larger than a few tens of centimetres). The mission is designed to get a global full coverage of these parameters. Near-real time transmission of the data to ground station centres, will be used to estimate geophysical parameters which will feed data assimilation into numerical models and forecast procedures.

\section{THE SWIM INSTRUMENT}

SWIM is designed to measure spectral properties of ocean waves based on the Jackson et al.'s concept [1] and used since the 90's on airborne systems [2,3]. The principle is based on the analysis of backscatter modulation due to the tilt of long waves, which is maximum when the look direction is parallel to the propagation direction of the waves. Unlike observations from 
SAR, the modulation is integrated over the azimuth dimension of the footprint, and the discrimination in azimuth is obtained through an azimuth scanning. First ideas of designing such a system for a satellite configuration were presented by [4], and first detailed specifications of a satellite mission was proposed by [5].

SWIM is a modified version of the design proposed in [5], now developed as part of the CFOSAT mission. SWIM will operate in Ku-band $(13.575 \mathrm{GHz})$, pointing at incidence angles between 0 and $10^{\circ}$ (multi-incidence beams) with a beam scanning and in azimuth $\left(0-360^{\circ}\right)$.

The 6 to $10^{\circ}$ beam are designed specifically for measuring directional ocean wave spectra in the wavelength range [70, 500 $\mathrm{m}$ ] at a scale of about $70 \mathrm{~km} \times 70 \mathrm{~km}$ on a $180 \mathrm{~km}$ wide swath with a minimum detectable wavelength of about $70 \mathrm{~m}$ and an angular resolution better than $15^{\circ}$.

The nadir looking beam is designed to measure wind speed and significant wave height with a concept and accuracy similar to standard altimeter missions (e.g.similar to JASON specifications).

All the beams ( 0 to $10^{\circ}$ ) will also be used to derive properties of the wave slope statistics from the profiles of radar cross-section.

A scheme of the geometry of observation is shown in Figure 1. For an orbit altitude of $500 \mathrm{~km}$, the footprint will be about $18 \mathrm{~km} \times 18 \mathrm{~km}$. In the look direction, the signal is analyzed with a high range resolution. The footprint will sweep a pseudo-circle with a diameter ranging from 18 to $90 \mathrm{~km}$ for incidence angles ranging from 2 to $10^{\circ}$. The surface pattern described by the instrument for all the incidence angles is shown in Figure 2 for successive scans over $360^{\circ}$ in azimuth, and for a satellite motion of $7 \mathrm{~km} / \mathrm{s}$. The number of elementary measurements (prior to averaging processes) is represented by the colour code. As the feed horns of the antenna will not be aligned on the antenna baseplate, switches of $90^{\circ}$ in azimuth view angles occur between some beams, leading to this ground pattern. Nonetheless, at the scale of a resolution cell $(70 \mathrm{~km}$ along-track), all the orientations are covered.

The acquisition durations spent on each incidence angle are called cycles. The global incidence coverage $\left[0^{\circ}-10^{\circ}\right]$ lasts a "macro cycle" of $218 \mathrm{~ms}$.

The transmitted signal is a chirp, a linearly frequency modulated pulse, with a $320 \mathrm{MHz}$ bandwidth and a $50 \mu$ s time length. After reception, the signal is compressed in range onboard, using numerical compression, allowing to reach a range resolution of about $47 \mathrm{~cm}$. Besides, each beam's PRF (Pulse Repetition Frequency) is selected in order to maximize the number of independent echoes, under some constraints such as the duty cycle of the high power amplification subsystem and the $\mathrm{Tx} / \mathrm{Rx}$ chronogram. The pulses acquired during each cycle are averaged in time and in range in order to reduce speckle and thermal noise. For data downlink constraints, some averaging (in time and range) has to be performed on-board.

The algorithm for signal time-integration will compensate in real-time for migrations due to range variations of a surface cell from one pulse to the other due to satellite advection and antenna rotation [6].

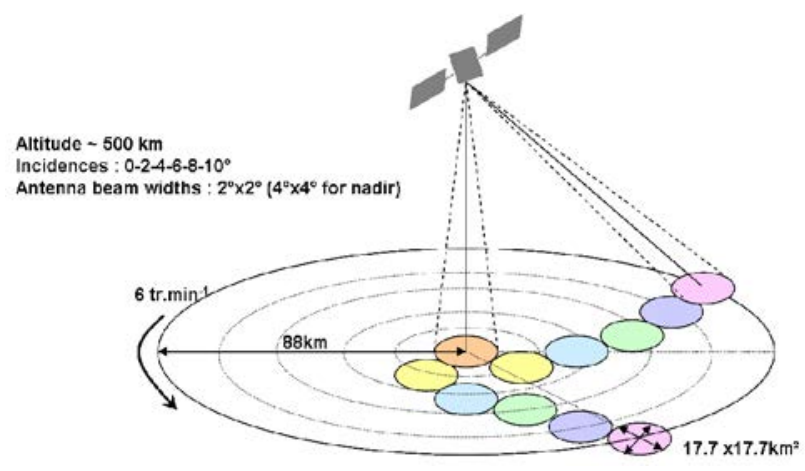

Figure 1: Geometry of observation of the SWIM instrument

For the off-nadir beams $\left(6\right.$ to $\left.10^{\circ}\right)$, the raw data transmitted to the ground stations will consist in the mean wave form computed in real-time with range migration compensation: signal intensity versus range with a range resolution of about $1.5 \mathrm{~m}$ integrated in time over 30 to $35 \mathrm{~ms}$ depending of the beam incidence. The geophysical products (directional wave spectra) will be computed at ground.

The validity of the principle to derive the spectra of ocean waves from this concept has been demonstrated several times using airborne systems developed at NASA (ROWS system, [2]) and in France (RESSAC radar, [3]). Simulation approaches have been used first to assess the feasibility of the concept [5] and are presently extended and used to perform detailed analysis of the system performance [7].

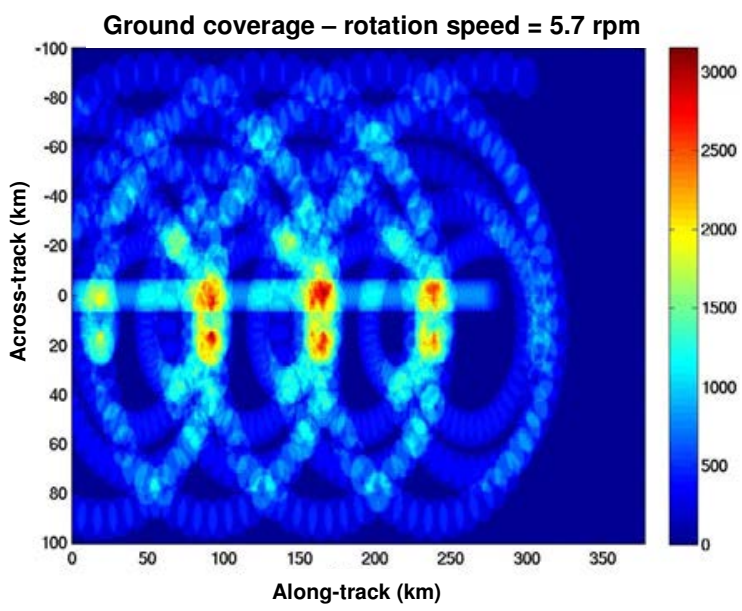

Figure 2: SWIM sampling at the surface.

The retrieval of the directional spectrum of surface waves is based on the spectral analysis of the radar backscatter modulations in each look direction, providing a modulation spectrum versus wave number of the ocean waves. After taking 
into account noise corrections (in particular speckle noise), and normalization factors, the modulation spectrum is related through a linear relationship to the slope spectrum of the waves, $\left.k^{2} F(k, \phi)\right)$ where $F(k, \phi)$ is the wave height spectrum, $k$ the wavenumber of the waves and $\phi$ the look direction. The azimuth scanning provides the complete information (directional spectrum), with however a $180^{\circ}$ ambiguity in the propagation direction. Overlap of the scans of the $6,8,10^{\circ}$ beams will be used to estimate directional wave spectra within surface boxes of about $70 \mathrm{~km} \times 70 \mathrm{~km}$.

The nadir pointing mode will be used to estimate significant wave height and wind speed as in conventional altimeter missions.

The 0 to $10^{\circ}$ radar mean cross-sections will be used to estimate statistical properties of wave slopes (short scales) as inspired by $[8,9]$

\section{SIMULATION OF PERFORMANCE OF WAVE SPECTRA ESTIMATES}

A simulation tool, called SimuSWIM, has been developed to derive the quality estimation of waves from the SWIM characteristics. SimuSWIM is an end-to-end simulator whose main steps are:

-simulation of the sea surface knowing analytical wave spectrum,

-computation of measured backscattered intensity,

-signal inversion to estimate wave spectrum,

-comparison of the estimation with the input spectrum.

Figure 3 proposes two examples of a theoretical spectrum used as input and the corresponding estimated one, taking into account the up-to-date SWIM definition. The results are accurate as they are very close to the theoretical spectrum used as input.

The modulation spectrum is a random variable. Thus, in [7], the expression of the theoretical standard deviation $\sigma$ has been derived.

$\sigma_{\hat{P}_{m}}(k)=\frac{1}{\sqrt{N_{s} L_{k}}} \sqrt{\frac{1}{N_{i m p}^{2}} \frac{P_{s p}^{2}(k)}{R^{2}(k)}+\frac{2}{N_{i m p}} \frac{P_{s p}(k) P_{m}(k)}{R(k)}+\frac{1}{N_{i m p}^{2} L_{d i s}^{2}} \frac{P_{b}^{2}(k)}{R^{2}(k)}}$

with $\mathrm{P}_{\mathrm{sp}}$ the speckle spectrum, $\mathrm{P}_{\mathrm{m}}$ the modulation spectrum, $\mathrm{R}$ the impulse response, $\mathrm{P}_{\mathrm{b}}$ the thermal noise spectrum, $\mathrm{N}_{\mathrm{imp}}$ the number of impulses averaged in time, $L_{d i s}$ the number of samples averaged in space, $\mathrm{N}_{\mathrm{s}}$ the number of spectrum averaged, $\mathrm{L}_{\mathrm{k}}$ the number of samples averaged in wavenumber.

This enables to define the $3 \sigma$ margins around the mean value. The estimation (Fig. 3) is quite entirely within these margins. Nonetheless, the simulation itself induces some artifacts due to digitalization and under-sampling. This may explain why some values are outside the $+/-3 \sigma$ margins.

Figure 4 illustrates the simulation on the $2 \mathrm{D}$ spectrum in a mixed sea case (non fully-developed wind sea spectrum and swell) compared to the reference. As automatic comparisons are not easy, some analysis tools are under development to automatically partition the spectrum into main sea states and calculate the associated geophysical parameters such as propagation direction, dominant wavelength, energy, etc.

Until now, theoretical analyses and simulations have confirmed that SWIM design is compliant with scientific requirements.

These results are based on two criteria calculated from the simulated modulation spectrum, $\hat{P}_{m}:$ i) a wavenumberdependent criteria, $\varepsilon(\mathrm{k})$, and ii) an integrated criterion $\Delta \mathrm{E}$ :

$\varepsilon(k)=\frac{\bar{\sigma}_{\hat{P}_{m}}(k)}{P_{m}(k)}$

$\Delta E=\mid \frac{E}{E_{\text {ref }}}-1$

where $E=\int_{k_{\min }}^{k_{\max }} \frac{\hat{P}_{m}(k, \phi)}{k} d k$

and $\mathrm{E}_{\mathrm{ref}}$ is computed with analytical shape of $\mathrm{P}_{\mathrm{m}} \cdot \hat{P}_{m}$ is the estimated modulation spectrum. The computation of $\Delta \mathrm{E}$ is made for a worthiest case, i.e. assuming that the estimation is equal to the reference spectrum $\mathrm{P}_{\mathrm{m}}$ plus the standard deviation:

$\hat{P}_{m}=\bar{\sigma}_{\hat{P}_{m}}+P_{m}$.

Figure 5 shows an illustration of these two criteria for the case of a wind sea modeled by a Pierson-Moskowitz function (and a wind speed of $13 \mathrm{~m} / \mathrm{s}$ ). Figure $5 \mathrm{a}$ shows that when integrated over the energy containing part of the spectrum $(0.04$ to $0.12 \mathrm{rad} / \mathrm{m}$ ), the predicted error on the total energy is less than $15 \%$. Figure $5 \mathrm{~b}$ shows that for all wavenumbers within $\left[\mathrm{k}_{\min }, \mathrm{k}_{\max }\right]$, the predicted uncertainty is less than $10 \% .\left(\mathrm{k}_{\min }, \mathrm{k}_{\max }\right.$ are wave numbers corresponding to the half-power energy density with respect to the peak).

Table 2 presents a summary of performances obtained for a set of simulations run in different conditions of sea-state and for an optimal set of instrument and processing parameters (parameters affecting the radar signal, $\hat{P}_{m}$, and its variance). The most important parameters affecting these estimates are the averaging coefficients and the Signal to noise ratio. They are linked to the initial spatial resolution (i.e. the emitted signal bandwidth), the sensor noises and the resolution requirements. According to these simulations, the instrument and processing characteristics chosen for SWIM are appropriate to measure spectral properties of wave in a large variety of conditions.

\section{ONGOING STUDIES RELATED TO SWIM AND CFOSAT}

Several French groups are presently working on the CFOSAT/SWIM preparation. 
First assimilation studies have been performed at MeteoFrance either using synthetic wave spectra or ENVISAT ASAR level 2 wave data [10]. The main conclusion is that the assimilation of directional information into wave prediction models induces a significant improvement in the estimate of wave parameters, such as the significant wave height and mean wave period. Figure 6 illustrates the impact of assimilation during the forecast period. It shows the index of assimilation, which characterizes the reduction of rms error on significant wave height gained through the assimilation, compared to Jason-1 observations. It shows that the impact of assimilation is larger (higher index of assimilation) and stays longer when directional observations are assimilated.. More generally, the assimilation system corrects well the misfit of the wave model in case of severe storm generating high swell travelling through the ocean. .

Developments of swell tracking algorithms have been performed and applied on SAR wave mode of ENVISAT [11] . They illustrate the interest of spectral wave observations to improve our knowledge of physical processes impacting swell propagation $[12,13]$. Such approaches will also be possible for SWIM data analysis.

In order to estimate directional spectra of ocean waves using the specific sampling geometry of SWIM (see Figure 2), work is under progress to prepare level 2 products based on a the multi-beam observations.

Simulations of cloud water and rain impacts on the signal are also under development to prepare rain flags.

\section{CONCLUSION}

SWIM is a new concept for measuring spectral properties of ocean waves from satellite. It is presently under development (phase B realization). It shall provide real breakthroughs in the wave observation field by providing wave spectra under large range of sea-state conditions. SWIM and SCAT combined on the same platform (CFOSAT) will foster new studies on coupled wind/wave phenomena and processes at the air/sea interface affected by wind and/or winds. The mission will be beneficial to operational forecast centers for sea-state forecast.

\section{REFERENCES}

1. Jackson, F. (1981). An analysis of short pulse and dual frequency radar techniques for measuring ocean wave spectra from satellites. Radio Science, 16(6) :1385-1400.

2. Jackson, F. C., W.T.Walton, and C.Y. Peng, A comparison of in situ and airborne radar observations of ocean wave directionality, Journal of Geophysical Research, 90(C1), 1005-1018, 1985

3. Hauser, D., G. Caudal, G.J. Rijckenberg, D. Vidal-Madjar, G. Laurent, and P. Lancelin, RESSAC: A new airborne FM/CW radar ocean wave spectrometer, IEEE Trans. Geosci. Remote Sensing 30 (5), 981-995, 1992

4. Jackson F. C., T.W. Walton, and P.L. Baker, Aircraft and satellite measurement of ocean wave directional spectra using scanning-beam microwave radars, Journal of geophysical research, Vol. 90, No. C1, Pages 987-1004, January 20,1985

5 Hauser D., E. Soussi, E.,Thouvenot, L. Rey: SWIMSAT: A real aperture radar to measure directional spectra of ocean waves from space, Main characteristics and performance simulation, Jour. Atmos. and Oceanic Tech, vol 18 No3, 421-437, 2001.

6. V. Enjolras V., L. Rey, L. Cros, S. Pouyez, T. Amiot, C. Tison, P. Castillan SWIM: a state-of-the art multi-incidence beams Ku-band waves scatterometer to go beyond current radar systems, IGARSS'09, July 2009.

7. C. Tison, T. Amiot, J. Bourbier, D. Hauser, V. Enjolras, L. Rey, P. Castillan, A spaceborne radar for directional wave spectrum estimation: first performance simulations, IGARSS'09, July 2009

8. Chapron, B., V. Kerbaol, D. Vandemark, and T. Elfouhaily (2000), Importance of peakedness in sea surface slope measurements and applications, J. Geophys. Res., 105(C7), 17195-17202.

9. Hauser D., G. Caudal, S. Guimbard, et A. Mouche, A study of the slope probability density function of the ocean waves from radar observations, J. Geophys. Res, 113, C02006, doi:10.1029/2007JC004264, 2008

10. Aouf, L., Lefèvre, J-M., Chapron, B. and Hauser, D. Recent improvements of the assimilation of upgraded ASAR Level 2 wave spectra in the wave model. Proceedings of the SEASAR 08, Frascati, January 2008.

11. Collard F., F. Ardhuin, B. Chapron (2009), Monitoring and analysis of ocean swell fields from space: New methods for routine observations, J. Geophys. Res., 114, C07023, doi:10.1029/2008JC005215.

12.Collard, F., F. Ardhuin, and B. Chapron, Persistency of ocean swell fields observed from space," J. Geophys. Res., vol. submitted, 2008.

13. Ardhuin F.,Chapron B, Collard F., Observation of swell dissipation across oceans, Geophysical Research Letters, March 2009 ; Volume 36 (L06607) : P. 1-5

\begin{tabular}{|c|c|c|}
\hline Sea states & $\begin{array}{l}\text { Estimation error }(\varepsilon) \\
\text { per } \mathrm{k} \text { on the } 3 \mathrm{~dB} \\
\text { wavelength domain }\end{array}$ & $\begin{array}{l}\text { Energy error } \\
(\Delta \mathrm{E}) \text { on the } 3 \mathrm{~dB} \\
\text { wavelength } \\
\text { domain }\end{array}$ \\
\hline $\begin{array}{l}\text { Pierson-Moskowitz } \\
\mathrm{U}=13 \mathrm{~m} . \mathrm{s}-1\end{array}$ & $\sim 10-15 \%$ & $\sim 10 \%$ \\
\hline $\begin{array}{l}\text { Pierson-Moskowitz } \\
\mathrm{U}=10 \mathrm{~m} . \mathrm{s}-1\end{array}$ & $\begin{array}{llll}15-20 & \% & (20 \% & \text { for } \\
k<0.06) & & & \\
\end{array}$ & $\sim 15-20 \%$ \\
\hline \begin{tabular}{|l} 
Jonswap \\
$\mathrm{U}=13 \mathrm{~m} . \mathrm{s}-1$
\end{tabular} & $-5 \%$ & $-5 \%$ \\
\hline $\begin{array}{l}\text { Jonswap } \\
\mathrm{U}=10 \mathrm{~m} . \mathrm{s}-1\end{array}$ & $-5 \%$ & $-5 \%$ \\
\hline Swell, Hs=4m & $\sim 10 \%$ & $\sim 10 \%$ \\
\hline Swell Hs $=2 \mathrm{~m}$ & $\sim 15 \%$ & $\sim 15 \%$ \\
\hline Swell Hs=1m & $20 \%$ & $20 \%$ \\
\hline
\end{tabular}

Table 1- Theoretical analysis of SWIM performance for different sea-state conditions. 


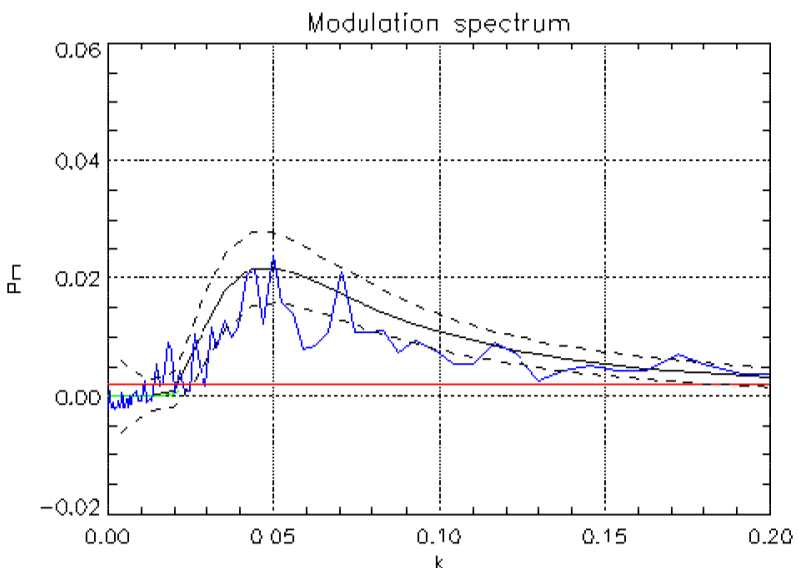

(a) Wind sea - Pierson-Moskowitz $U=13 \mathrm{~m} / \mathrm{s}$

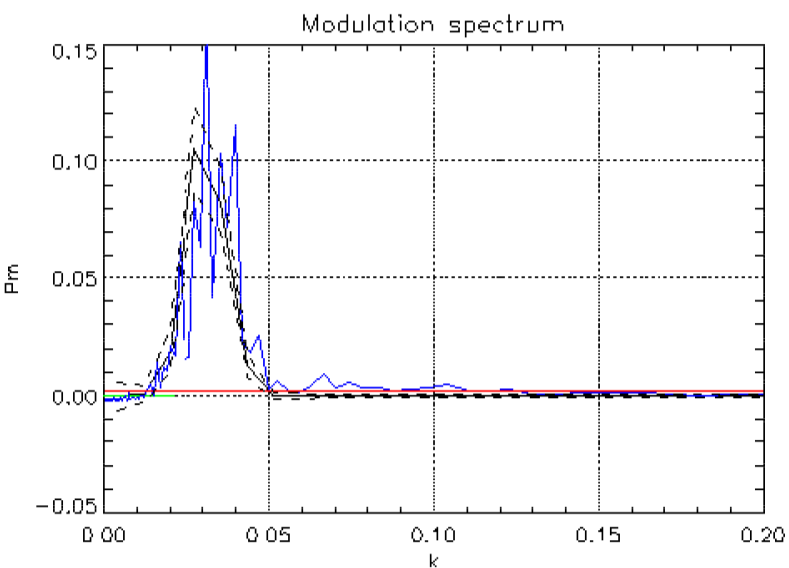

(b) Swell $-\mathrm{Hs}=4 \mathrm{~m}-\mathrm{U}=10 \mathrm{~m} / \mathrm{s}$

Figure 3. Simulation of two sea states (wind sea (a) and swell (b)). The black curves are the input spectrum, the blue curves the estimated one taking into account all the properties of the instrument and of the processing chain, the red curves the speckle spectrum. The dotted lines are the +/-36 margin around the theoretical value ( $\sigma$ begin the standard deviation).

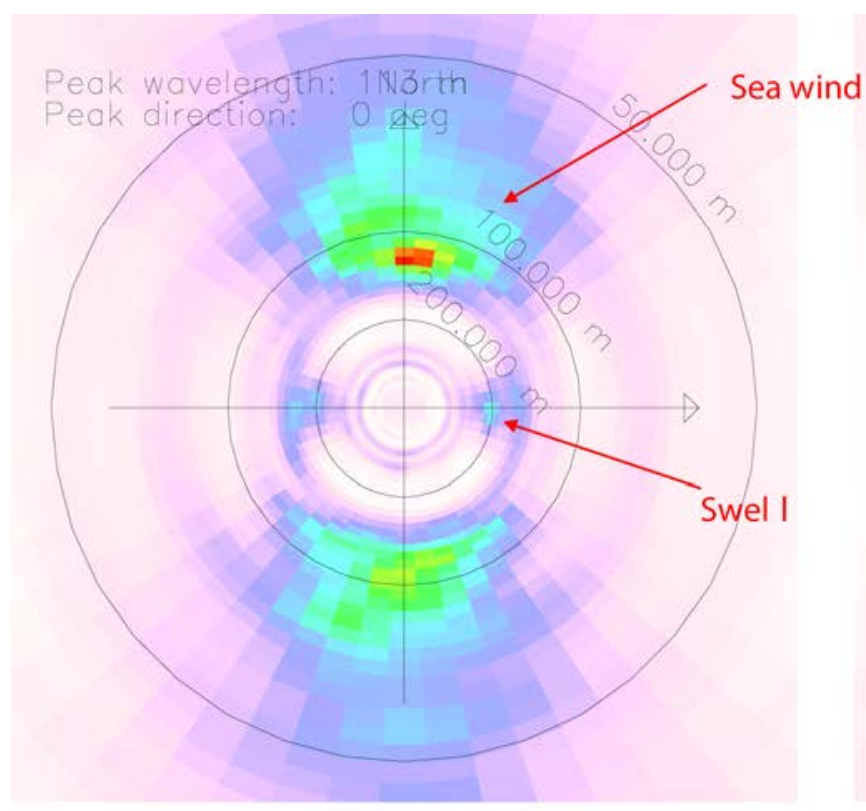

Estimated 2D spectrum

Jonswap $\mathrm{U}=10 \mathrm{~m} \cdot \mathrm{s}-1$ Fetch $=60 \mathrm{~km}$

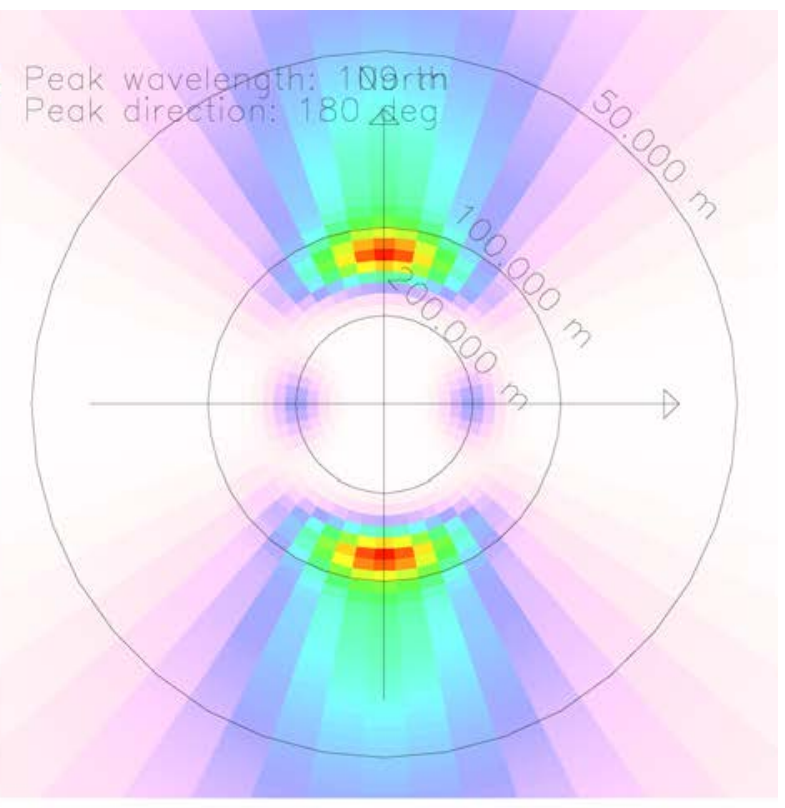

Reference 2D spectrum

$\Phi=0-$ Swell $\mathrm{Hs}=1 \mathrm{~m} \quad \Phi=90$

Figure 4. Simulated 2D wave spectra for a Jonswap spectrum $\left(U=10 \mathrm{~m} \cdot \mathrm{s}^{-1} \mathrm{fetch}=60 \mathrm{~km}\right)$ after inversion (left) compared to the analytical reference (right). The resulting peak wavelength and peak direction as estimated after partitioning are noted on the figure (left top). The $180^{\circ}$ ambiguity in direction is not removed in the example. 


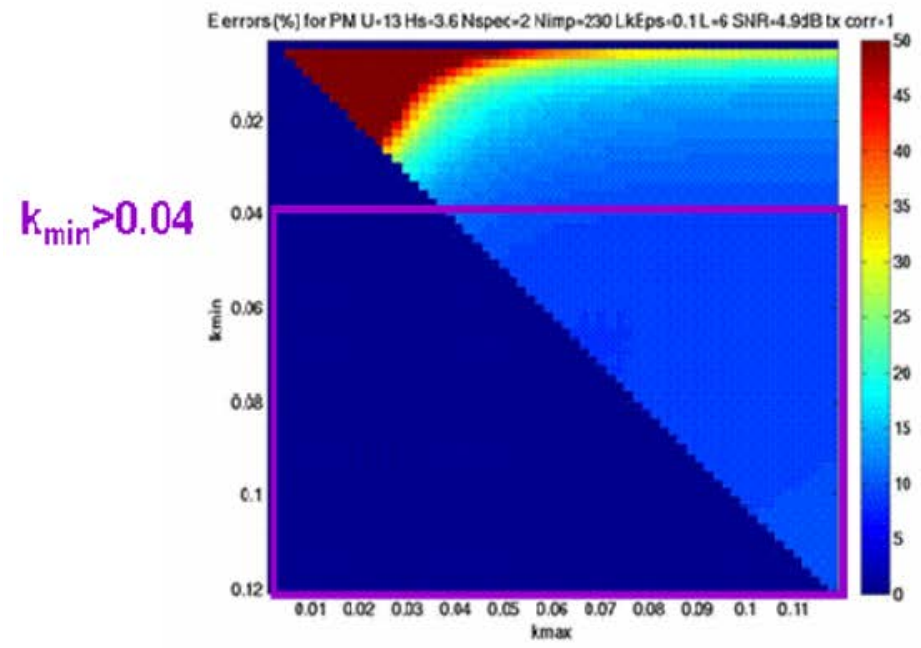

(a) $\Delta E$ for every $\left(k_{\text {ren }}, k_{\text {max }}\right.$ ) value

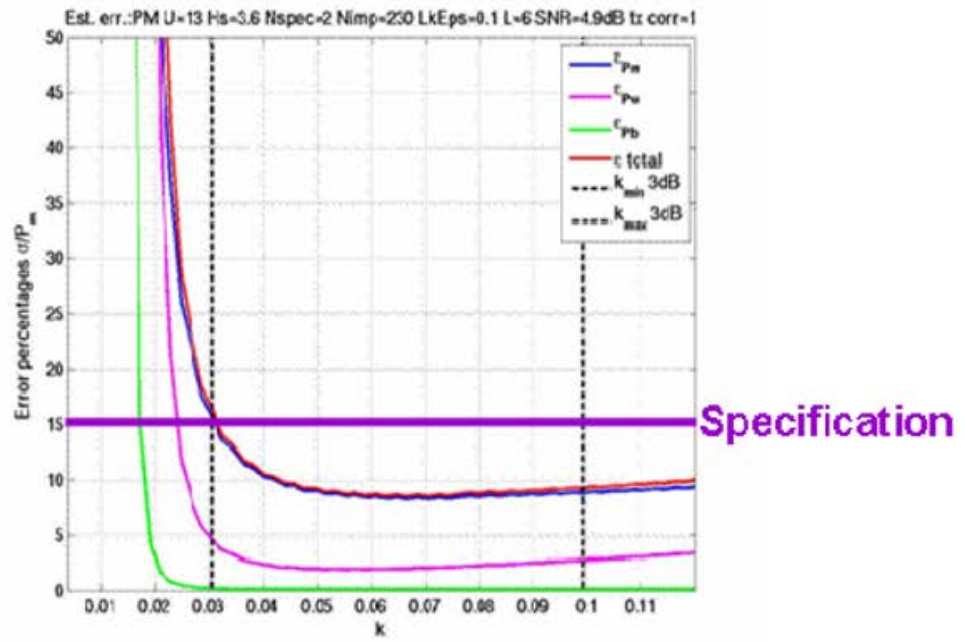

(0) $\varepsilon$

Figure 5. Quality criteria on the inverted modulation spectrum, in a wind-sea case (Pierson Moskowitz spectrum with a $13 \mathrm{~m} / \mathrm{s}$ wind speed). (a) uncertainty on integrated energy (b) estimation error per wavenumber (the red curve stands for the sum all of sources of errors)

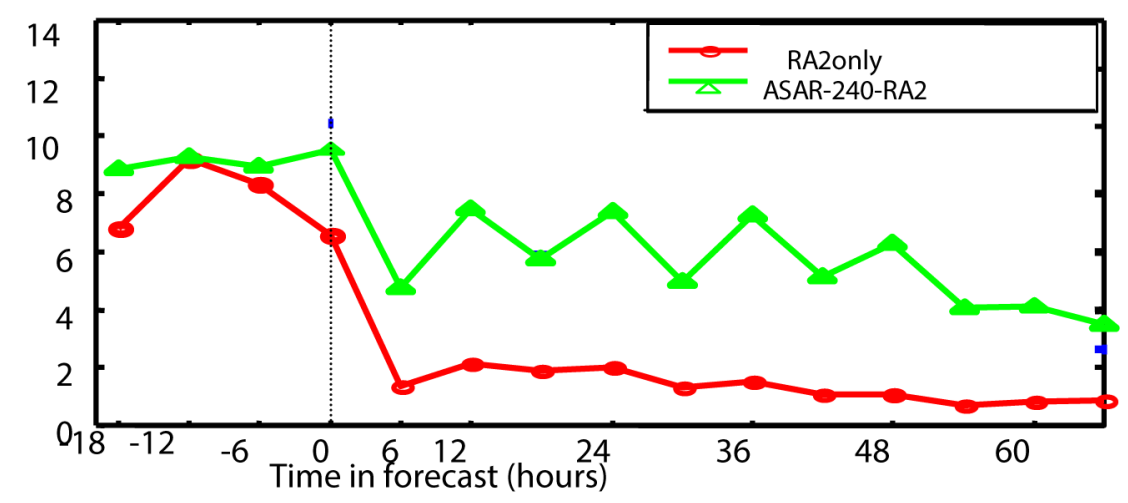

Figure 6: Index of assimilation impact versus time during the forecast period. Red line stands for the assimilation of altimeter Ra-2 wave height only, while green line indicates the assimilation of altimeter Ra-2 and ASAR wave spectra. 